\title{
Optimization of Extraction Techniques SPE, QuEChERS and Ultrasound Method for Trace level Determination of Imidacloprid in Vegetables (Cabbage and Spinach) and Soil by (UFLC)
}

\author{
Prakash Samnani $^{1 *}$ Kamlesh Vishwakarma ${ }^{2}$ and Bipul Bihari Saha ${ }^{2}$ \\ ${ }^{1}$ Department of Chemistry, Faculty of Science, M S University of Baroda, Vadodara, Gujarat, India \\ ${ }^{2}$ Nagarjuna Agrichem Limited (R\&D Center), Telangana, India
}

Received: August 03, 2015; Accepted: September 15, 2015; Published: October 03, 2015

*Corresponding author: Prakash Samnani, Department of Chemistry, Faculty of Science, The M S University of Baroda, Vadodara, India, Pin: 390 002; Email: pbsam2015@gmail.com

\begin{abstract}
A simple, sensitive, fast and inexpensive method was developed using solid-phase extraction (SPE), ultrasound and QuEChERS method with Ultra Fast Liquid Chromatography (UFLC) for trace level determination of imidacloprid in vegetables (Cabbage and Spinach) and Soil. The method was validated using Cabbage, Spinach and soil samples spiked with imidacloprid at different concentration levels (LOQ 0.01, $10 \times$ LOQ 0.10 and $50 \times$ LOQ $0.50 \mu \mathrm{g} / \mathrm{g}$ ). Average recoveries (using each concentration 5 replicates) ranged from 89.39 to $99.5 \%$, with relative standard deviations less than $2.15 \%$, calibration solutions concentration used were in the range $0.005-1.0$ $\mu \mathrm{g} / \mathrm{mL}$ and limit of detection (LOD) and limit of quantification (LOQ) were $0.005 \mu \mathrm{g} / \mathrm{mL}$ and $0.01 \mu \mathrm{g} / \mathrm{mL}$, respectively.
\end{abstract}

Keywords: Solid-Phase Extraction; Quechers; Imidacloprid; Method Validation; UFLC

\section{Introduction}

Imidacloprid [IUPAC name 1-(6-chloro-3- pyridylmethyl)$\mathrm{N}$-nitroimidazolidin-2- lideneamine] is a common pesticide used in India, belonging to a chemistry regime (chloronicotinyl insecticide). Pesticides constitute one of the most hazardous groups of contaminants to human health, fauna and environment. The majority of such substances are applied directly to soil or sprayed over crop fields and hence are released directly to the environment. The pesticide residue analysis is important to know how much pesticide remains in fruits and vegetables. Fruits and vegetables are important components of the human diet since they provide essential nutrients that are required for most of the reactions occurring in the body. Pesticide residue analysis in vegetables and food has traditionally been performed using gas chromatography (GC), but there is increasing use of liquid chromatography (LC). LC is favored for polar, less thermally-stable and less volatile compounds. GC and GC-MS are preferred for volatile and thermally-stable species. The data quality can be improved through better retention and separation of components, especially for structurally similar pesticides and high-level matrix co-extractives.

A number of methods have been employed to measure imidacloprid residues: Photochemical fluorimetric method [1], electrochemical method, enzyme-linked immune sorbent assays [2], capillary electrophoresis, gas chromatographymass spectrometry (GC-MS) and High performance liquid chromatography HPLC [3]. Among these methods GC and LC are the most suitable methods. However, GC cannot be used directly to determine imidacloprid due to the poor volatility and polarity. Imidacloprid residues can be analyzed by derivatization and gas chromatography. In contrast to GC, HPLC is more effective and appropriate for the residual analysis of imidacloprid, and it has been successfully employed for assaying imidacloprid in the soils, water as well as in the vegetables [4]. High performance liquid chromatography (HPLC) appears to be a suitable alternative because of the thermolability and polarity of imidacloprid. The LC method gave good results for imidacloprid in groundwater, soils, fruits and vegetables but the limit of detection (LOD) was still too high for the present purpose [5]. Furthermore, the required method must satisfy strict quality criteria to be classified as a trace levels determination and low levels extraction procedure. Prakash Samnani, et al. [6] has been reported a simple and sensitive method for imidacloprid using HPLC and extraction procedure for imidacloprid which is a traditional method. The ultra fast liquid chromatography (UFLC) is quite popular for enhancing the laboratory's productivity. The combination of Prominence UFLC and new reversed-phase columns achieves ten times higher speed compared to a conventional HPLC system. Furthermore, a high-speed sample injection mechanism significantly reduces total analytical cycle time. With this combination, UFLC offers three times higher resolution than conventional HPLC so that the possibility of analysis is increased for samples that are not sufficiently separated by conventional HPLC. The UFLC yields good resolution, low sensitivity and fast analysis with area repeatability better than conventional HPLC. 
The improvement in analysis is largely due to use of smaller particles used in the column ( $3.5 \mu \mathrm{m}$ columns) and associated hardware to achieve higher pumping pressures, supported by faster injections and detection.

QuEChERS (Quick-Easy-Cheap-Effective-Rugged-Safe) is a sample preparation approach developed by Anastassiades, et al. [7] as a simple, rapid, effective, yet inexpensive, way to extract pesticide residues from fruits and vegetables, followed by a dispersive solid phase extraction (dSPE) cleanup of the extract. It is well established that QuEChERS can result in good recovery values not only for a large number of pesticides but also for a wide variety of other compounds. Kapoor U, et al. [8] have reported QuEChERS (quick, easy, cheap, effective, rugged, and safe) method of extraction procedure for imidacloprid in fruits, fruit juices, and baby foods followed by high-performance liquid chromatographic analysis, and imidacloprid residues were qualitatively confirmed by liquid chromatography-mass spectrometry. We developed a new extraction scheme with high recovery rates, coupled to a UFLC methodology in order to extract and quantify imidacloprid in soils, vegetables (cabbage and spinach). The present study will concern extensively to trace level determination of imidacloprid in vegetables (cabbage and spinach) and soil by validating and using Quick, Easy, Cheap, Effective, Rugged and Safe (QuEChERS) and solid phase extraction method and ultrasound method followed by Ultra Fast Liquid Chromatography (UFLC). We modify the (QuEChERS) method for determination of imidacloprid. Comparison between (QuEChERS) extraction method, Solid phase extraction and ultrasound for its efficiency and sensitivity were carried out. More generally, such a method can be easily adapted for the analysis of fruits and vegetables.

\section{Materials and Methods}

Analytical reference standards of imidacloprid (98.5\% purity) were obtained from Dr. Ehrenstorfer, Germany. All the other chemicals and solvents used were analytical and HPLC grade.

\section{Samples}

Different types of agricultural products (e.g. cabbage and spinach) were purchased from local markets in Hyderabad, India. Samples were ground with a high speed grinder in 2 liter capacity jar with a lid and stored at $-20 \pm 2^{\circ} \mathrm{C}$.

\section{Standard preparations}

Stock solution: Reference (stock) standard solutions (1000 $\mu \mathrm{g} / \mathrm{mL}$ ) of Imidacloprid were prepared in acetonitrile. Stock solutions were kept at $-20 \pm 2{ }^{\circ} \mathrm{C}$.

Calibration mixture solution: Calibration mixtures of concentration levels $0.005,0.01,0.05,0.1,0.5$ and $1.0 \mu \mathrm{g} / \mathrm{mL}$ were prepared in acetonitrile by diluting suitable aliquots of stock solution in acetonitrile and were stored at $-20 \pm 2{ }^{\circ} \mathrm{C}$.

\section{Instrumentation}

Quantitative analysis was performed on a quaternary gradient UFLC with Shimadzu prominence UFLC LC-20AD pumps, with a $20 \mu \mathrm{l}$ sample and UV detector. The output signal was monitored and integrated using Shimadzu LC solution software. An Agilent C18 column (100 mm x 4.6mm i.d 3.5 $\mu \mathrm{m}$ ) was used for separation. Mobile phase A: $0.01 \%(\mathrm{v} / \mathrm{v})$ acetic acid in water (60) and Mobile phase B: acetonitrile (40). The mobile phase was delivered to $1 \mathrm{~mL}$ flow rate and detector set of $252 \mathrm{~nm} \lambda$ max was used for analysis. Imidacloprid standard showed a sharp peak at 4.93 minutes under the described UFLC conditions.

The Figures 1, 2 and 3 depict a typical chromatogram of the separation of imidacloprid reference standard and recovery in vegetable and soil spike samples. The vegetable samples were analyzed by LC-MS Ion Trap for imidacloprid peak confirmation and other matrix effects.

\section{Method Validation}

The specificity of the analytical method was studied by injecting solvent, reference standard solution, control soil and vegetable (cabbage and spinach sample) extracts onto Ultra Fast Liquid Chromatography (UFLC). There was no interference of the components with each other. The linearity was established by injecting five different concentrations, viz. 0.005 to $1.0 \mu \mathrm{g} / \mathrm{mL}$ and determining the response of imidacloprid, these were fitted by linear regression to assess the linearity. The limit of detection was determined to be $0.005 \mu \mathrm{g} / \mathrm{mL}$ at a level of approximately three times the background of control injection around the retention time of the peak of interest. Detection Limit (signal-tonoise ratio $=3 \pm 0.5: 1$ ) was established.

Ratio approach: This method can only be applied to analytical procedures which exhibit baseline noise. It is determined by comparing measured signals from samples with known low concentrations of analyte with those of blank samples and establishing minimum concentration at which the analyte can be reliably detected. A S/N ratio of 3:1 is considered acceptable for estimating LOD (with Relative Standard Deviation (RSD) $\leq 10 \%$ ) whereas for LOQ S/N ratio of 10:1 is considered appropriate (with Relative Standard Deviation (RSD) $\leq 3 \%$ ).

Standard deviation of the response and slope: The LOD and LOQ may be expressed as LOD $=3.3 \times \sigma / \mathrm{S}$ and LOQ $=10 \times \sigma / \mathrm{S}$ where $\sigma=$ the standard deviation of the response, $\mathrm{S}=$ the slope of the calibration curve of analyte. The slope $\mathrm{S}$ may be estimated from the calibration curve of the analyte. The value of $\sigma$ may be taken from as standard deviation of analytical background responses of an appropriate Three of blank samples. The linear dynamic range of imidacloprid is shown in Figure 5.

The precision (\% RSD) of the analytical method was determined by five replications in the duplicate injection of fortified substrate soil and cabbage and spinach sample extracts at LOQ, $10 \times$ LOQ and $50 \times$ LOQ levels. The accuracy (\% recovery) of the method was determined by five replications (in duplicate) injection of fortified substrate soil, cabbage and spinach sample extracts at LOQ, 10 times and 50 times LOQ levels. Precision (\% RSD) should not exceed $20 \%$ at lower levels.

\section{Sample fortification}

A representative sample $(10 \mathrm{~g})$ of soil and vegetables (cabbage and spinach) was transferred to Polyethylene (PFTE) 

(UFLC)

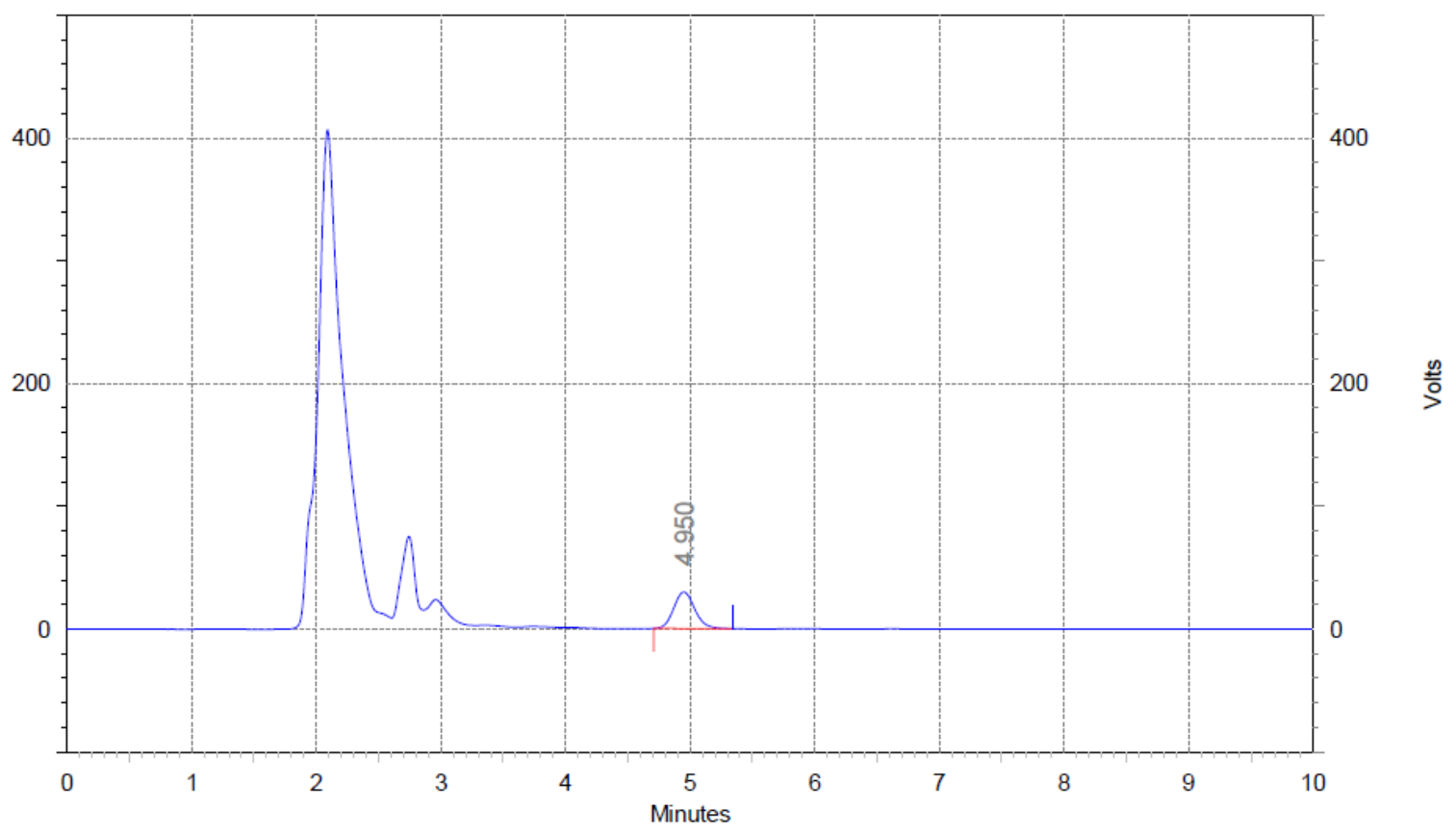

Figure 1: Chromatograms of Imidacloprid spike at $10 \mathrm{X} \mathrm{LOQ} \mathrm{Level} \mathrm{in} \mathrm{vegetable} \mathrm{sample} \mathrm{(spinach).}$

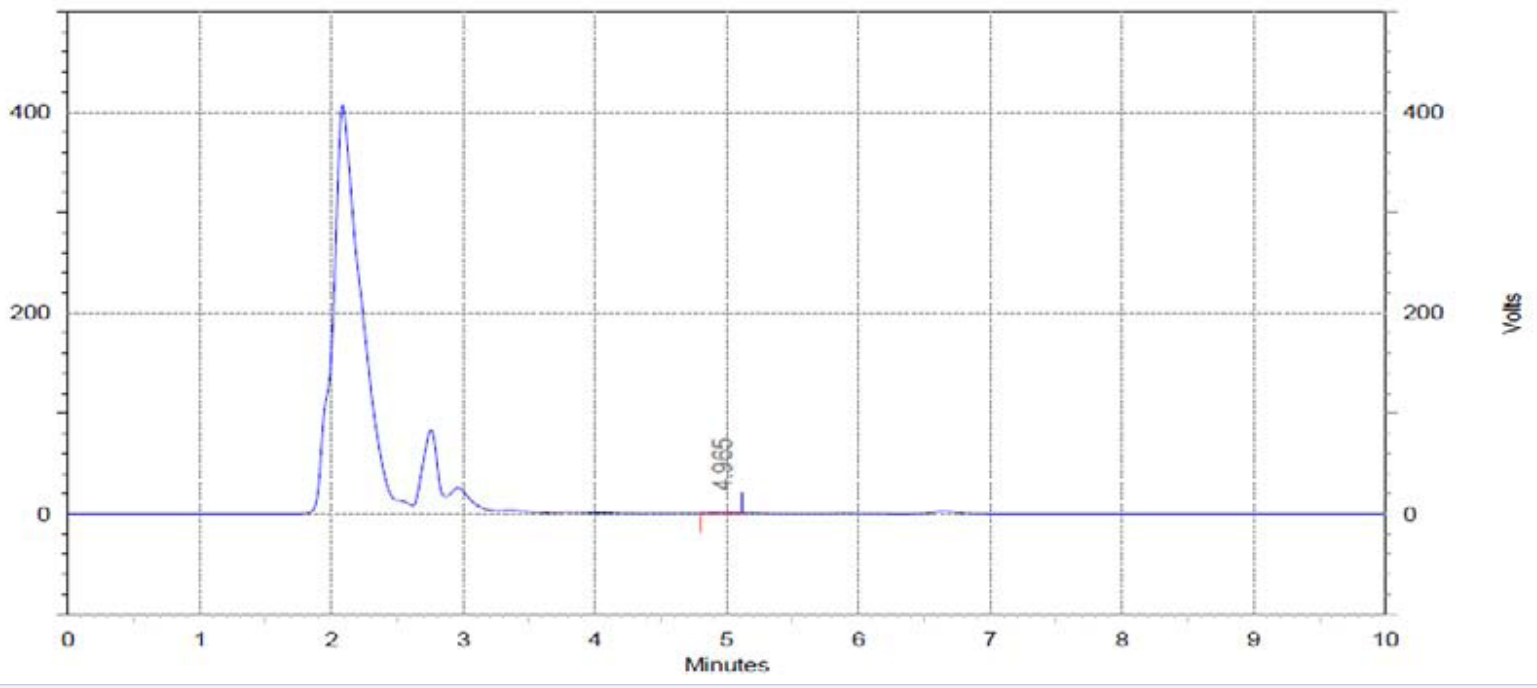

Figure 2: Chromatograms of Imidacloprid spike at LOQ Level in vegetable sample (spinach).

$50 \mathrm{~mL}$ tubes. The soil and vegetable sample was fortified with imidacloprid at three different fortification levels: LOQ and $10 \times$ LOQ and 50 LOQ levels, separately. The control samples were processed similarly where in acetonitrile was added.

\section{Extraction procedure}

Extraction procedures used in our study for analysis of Imidacloprid in vegetables (cabbage and spinach) and soil was solid phase extraction (SPE), QuEChERS and ultrasound method as described below:

QuEChERS method: A quantity of $10 \mathrm{~g}$ soil and vegetables samples (cabbage and spinach) was weighed and transferred in
Polyethylene (PFTE) $50 \mathrm{~mL}$ tube, then $20 \mathrm{~mL}$ acetonitrile was added and shaken vigorously for one minute, 2.0 gm anhydrous $\mathrm{MgSO}_{4}$ and 0.5 gm sodium chloride were then added and mixture shaken immediately for 1.0 minute. Centrifugation was carried out at $4000 \mathrm{rpm}$ for 5 minutes and cleanup was done with 25 mg PSA (Primary secondary amine). Primary Secondary Amine (PSA) has been found as the most effective sorbent for removal of various matrices and significantly reducing the matrixenhancement effect. On the other hand, graphitized carbon black (GCB) is very useful for the removal of coloring substances (i.e. pigments) and sterols. Therefore, PSA itself and/or combination of GCB/PSA dual layer should be most effective for sample 

(UFLC)

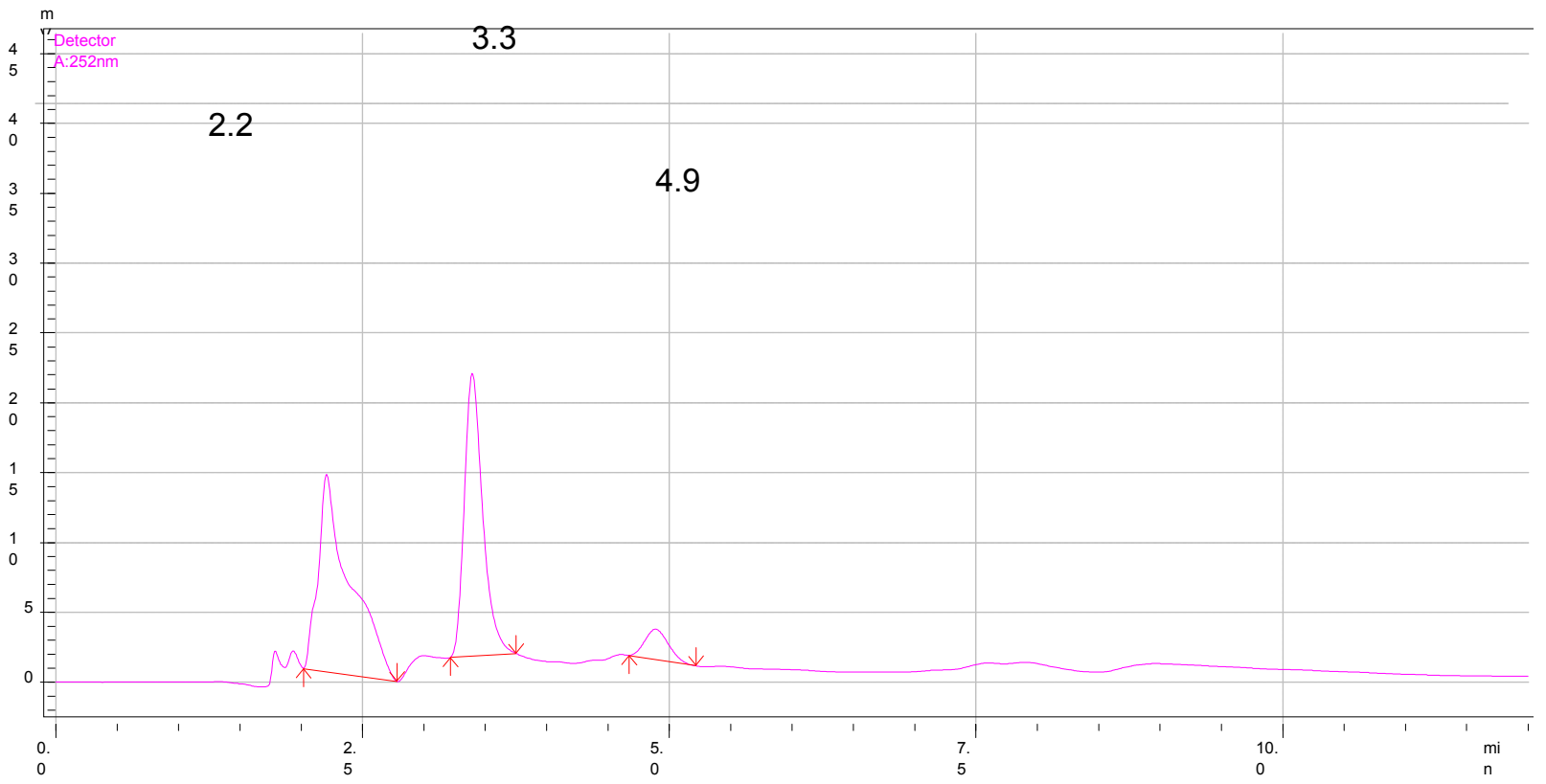

Figure 3: Chromatograms of Imidacloprid spike at LOQ Level in Soil samples.
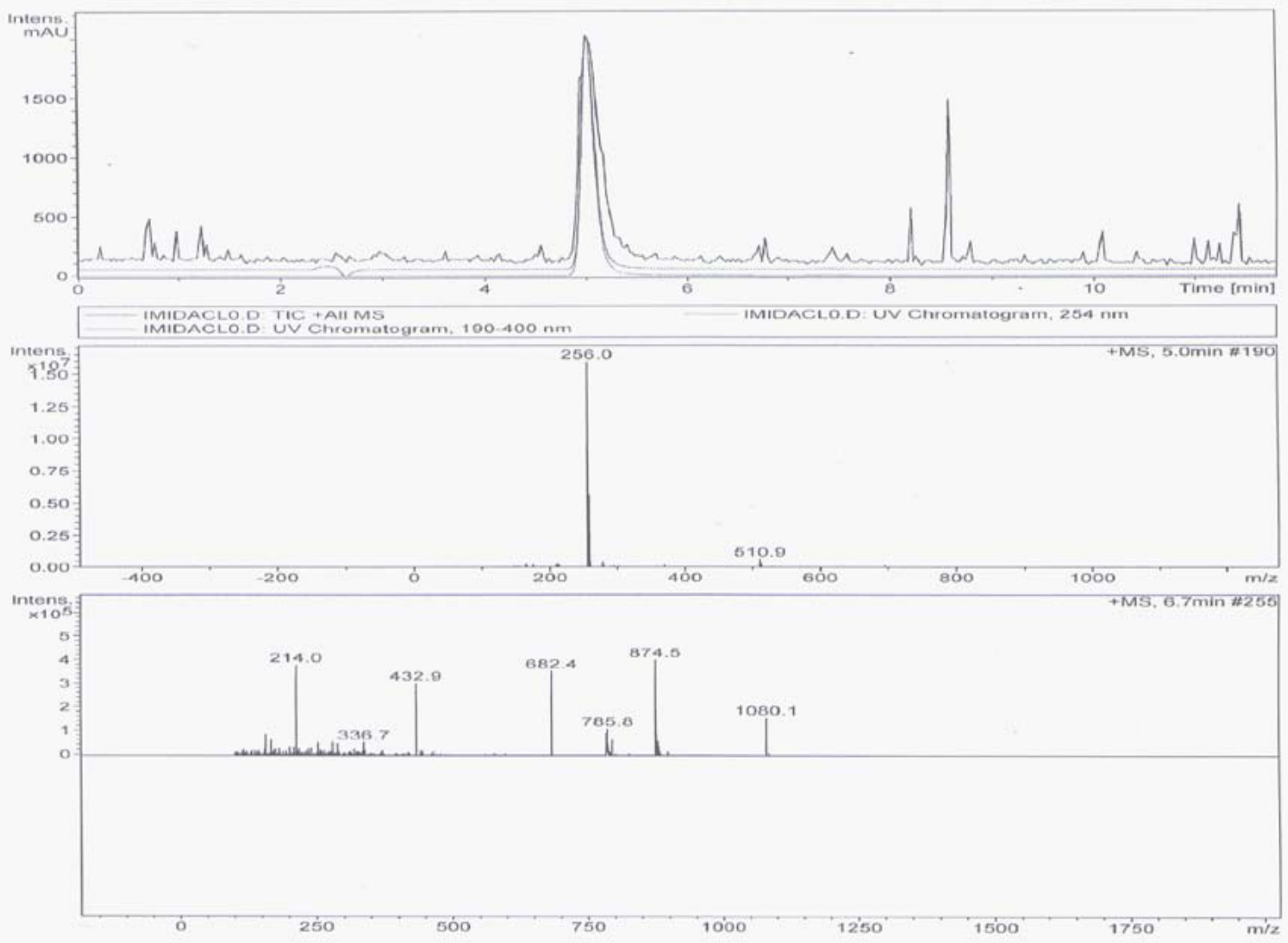

LCMS Report (A4-Opt2)

Page 1 of 1

Figure 4: Mass spectra of Imidacloprid spike soil samples. 
cleanup. Supernatant ( $4 \mathrm{~mL}$ ) of the clear solution was transferred to $5.0 \mathrm{~mL}$ centrifuge tube, $25 \mathrm{mg}$ PSA was added followed by 25 mg C18 and $200 \mathrm{mg}$ anhydrous $\mathrm{MgSO}_{4}$, mixture was shaken well and centrifuged at $3000 \mathrm{rpm}$. An aliquot was take out, dried using Buchi nitrogen evaporator and residue dissolved in $2.0 \mathrm{~mL}$ of mobile phase. The final samples were injected on UFLC.

Extraction procedure for imidacloprid with Ultrasound technique from vegetable samples: $10 \mathrm{~g}$ of vegetables sample (cabbage and spinach) was homogenized and was spiked at two levels 0.1 and $0.5 \mu \mathrm{g} / \mathrm{g}$, with imidacloprid. The fortified sample was mixed with $25 \mathrm{~mL}$ acetonitrile. This was placed on an ultrasound bath for 20 minutes and centrifuged at $3000 \mathrm{rpm}$ for 30 minutes. This was found to improve the extraction efficiency of the imidacloprid. This procedure was repeated twice and the extracts were pooled. A volume of $5.0 \mathrm{~mL}$ extract was taken for cleanup by solid phase extraction.

Solid Phase Extraction method (SPE): $10 \mathrm{~g}$ of soil sample was weighed in reagent bottles of $50 \mathrm{~mL}$ capacity. $20 \mathrm{~mL}$ acetonitrile was added and the bottle was shaken on an orbital shaker for 10 minutes. This process was repeated twice. The extract was filtered through Whatman filter paper No.1 into $100 \mathrm{~mL}$ graduated tubes. From this, $20 \mathrm{~mL}$ aliquot of the extract was concentrated to less than $2.0 \mathrm{~mL}$ on the N-EVAPTM. The concentrate was suspended in 2 to $3 \mathrm{~mL}$ of methanol. A BondElute cartridge was conditioned by rinsing with $5.0 \mathrm{~mL}$ of methanol on a vacuum and the extract suspension was loaded on the cartridge and the eluant was collected in a test tube. The test tubes were rinsed 2 times with $3 \mathrm{~mL}$ methanol. Each of the cartridge rinses was collected in the manifold. The eluate was concentrated to about $2.0 \mathrm{~mL}$ and final sample was made with methanol in $5 \mathrm{~mL}$. This sample was directly injected to reverse phase Ultra Fast Liquid Chromatography (UFLC).

\section{Instrumentation for LC-MS Ion Trap Instrument}

The market and method validation samples analyzed by LCMS for peak conformation. The quantification and analysis of samples using UFLC. The system was equipped with a binary solvent pump with the autosampler. The imidacloprid MS spectrum of the m/z 256 parent ion and daughter ion 214 is shown in figure 4 . The MS system was constituted of a standard atmospheric pressure ionization source and positive mode configured as ESI. The ion polarity is positive and capillary exit 109.3 volt. The dry temperature $350^{\circ} \mathrm{C}$ and nebulizer $50.00 \mathrm{psi}$ were used. The LC system was fitted with C18 column and mobile phase A: $0.01 \%(\mathrm{v} / \mathrm{v}$ ) formic acid in water (60) and Mobile phase $\mathrm{B}$ : acetonitrile (40) for samples analysis for peak conformation.

\section{Results and Discussion}

We have validated the analytical method and extraction procedure for the determination of trace amounts of imidacloprid in/on soil, cabbage and spinach, using Ultra Fast Liquid Chromatography (UFLC). The linearity of the detector response was tested for Imidacloprid, in a solvent and in matrix soil and vegetable extract over the concentration range of 0.005 to1.0 $\mu \mathrm{g} / \mathrm{mL}$. A very precise linear relation between the injected amount and the resulting peak area was observed over the entire concentration range with correlation coefficient value of 0.999 (Table 1 and figure 5). The accuracy and precision of the method were evaluated on the basis of the recoveries obtained for fortified soil and cabbage and spinach samples. The limit of quantization (LOQ) was found to be $0.01 \mu \mathrm{g} / \mathrm{g}$ in the soil, cabbage and spinach. The limit of detection (LOD) was $0.005 \mu \mathrm{g} / \mathrm{g}$. Recoveries for imidacloprid varied from 95.0 to $95.62,96.82$ to 99.52 and 90.34 to 97.02 for the used solid phase extraction procedure in soil, cabbage and spinach, respectively (Figures 1, 2 \& 3). Recoveries for imidacloprid varied from 95.0 to 95.62 and 89.39 to 94.41 used QuEChERS method procedure in cabbage and spinach, respectively. The accuracy (\% recovery) data in the soil, cabbage and spinach is depicted in Table 1. Ralf, et al. [9] have reported a gradient HPLC - tandem MS method for imidacloprid recovery. In their study, repeatability of the method was determined for the analyte by running a set of five recoveries each at two different fortification levels for selected matrices. The resulting mean recovery rates ranged from 79 to $104 \%$ with relative standard deviations between 0.8 and $15.3 \%$.

Similar results were found by us in present method validation for soil and vegetable samples. The repeatability of the method in the present study was determined for each fortification level by running a set of five recoveries each at different fortification levels for selected matrices. The \% RSD for the resulting mean recovery rates ranged from 94.00 to $95.62 \%$ in soil with relative standard deviations between $0.25 \%$ to $1.61 \%$. The $\%$ RSD for spinach and cabbage ranged varied from $0.87 \%$ to $2.15 \%$ and $0.89 \%$ to $1.00 \%$ in solid phase extraction method. In QuEChERS method \% RSD range varied from $1.02 \%$ to $1.94 \%$ and $0.14 \%$ to $1.13 \%$ Imidacloprid recovery for Ultrasound technique in vegetable samples of spinach recovery range varied from 92.36 to 96.84 with \% RSD 1.12 and $1.65 \%$. Similarly, cabbage sample recovery range varied from 94.945 to $95.32 \%$ with \%RSD was $0.98 \%$ to $1.40 \%$. In ultrasound extraction, procedure recovery is good but at lower fortification levels $0.01 \mu \mathrm{g} / \mathrm{g}$ recovery is low. The vegetable samples were analyzed by LC-MS Ion Trap for imidacloprid peak confirmation and another matrix effect (Figure 4). We found the imidacloprid parent ion $\mathrm{m} / \mathrm{z} 256$ in positive mode and very sharp peak and no any matrix effect.

\section{Conclusion}

The present study reports trace level determination of

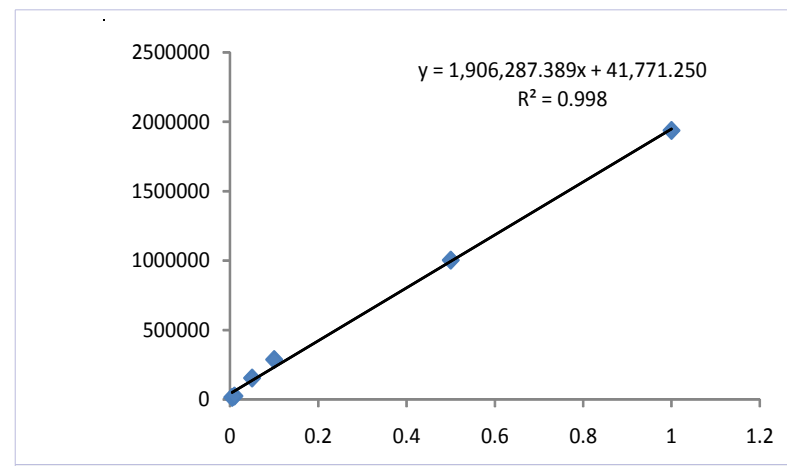

Figure 5: Linear Dynamic Range (LDR). 

(UFLC)

Table 1: Method Validation Results Summary for Imidacloprid.

\begin{tabular}{|c|c|c|c|c|c|c|c|}
\hline \multirow[b]{3}{*}{ Parameters } & \multicolumn{7}{|c|}{ Substrate } \\
\hline & \multicolumn{2}{|c|}{ Cabbage } & \multicolumn{2}{|c|}{ Spinach } & \multirow{2}{*}{\begin{tabular}{|l|} 
Soil \\
SPE \\
\end{tabular}} & \multirow{2}{*}{\multicolumn{2}{|c|}{$\begin{array}{l}\text { Cabbage Spinach } \\
\text { Ultrasound technique }\end{array}$}} \\
\hline & SPE & QuEChERS Method & SPE & QuEChERS Method & & & \\
\hline Specificity & \multicolumn{7}{|c|}{ No interference } \\
\hline $\begin{array}{l}\text { Fortification Levels } \\
(\mu \mathrm{g} / \mathrm{g})\end{array}$ & \multicolumn{4}{|c|}{ Precision (\%RSD) } & & & \\
\hline 0.01 & 1.71 & 1.02 & 1.00 & 0.14 & 0.25 & \multicolumn{2}{|l|}{-} \\
\hline 0.10 & 2.15 & 1.12 & 0.89 & 2.13 & 1.02 & 1.4 & 1.65 \\
\hline 0.50 & 0.87 & 1.94 & 0.96 & 1.13 & 1.61 & 0.98 & 1.12 \\
\hline \multicolumn{8}{|c|}{ Accuracy (\% Recovery) } \\
\hline 0.01 & 96.82 & 95.00 & 90.34 & 89.39 & 95.00 & \multicolumn{2}{|l|}{-} \\
\hline 0.10 & 96.39 & 94.00 & 93.13 & 94.41 & 94.00 & 95.32 & 96.84 \\
\hline 0.50 & 99.52 & 95.62 & 97.02 & 90.4 & 95.62 & 94.94 & 92.36 \\
\hline
\end{tabular}

imidacloprid in vegetables (cabbage and spinach) and soil by validating and using Quick, Easy, Cheap, Effective, Rugged and Safe (QuEChERS) extraction method and solid phase extraction followed by Ultra Fast Liquid Chromatography (UFLC). Comparison between (QuEChERS) extraction method and solid phase extraction and ultrasound technique for its efficiency and sensitivity has been carried out. QuEChERS) extraction method simple clean-up procedure using PSA was found to yield sufficiently clean samples. A quick, simple extraction procedure has been established for the determination of imidacloprid in vegetables (cabbage and spinach) and soil samples with good percentage recovery (89.39-99.52\%) in all cases. The limit of detection was $0.005 \mu \mathrm{g} / \mathrm{g}$. The satisfactory validation parameters such as linearity, recovery, precision and very low limits were obtained and were according to the SANCO guidelines [10]. QuEChERS method was suitable for market samples analysis. It has been proved that the proposed method and extraction procedure provides a good sensitivity; reproducibility and recovery (Table 1). The results demonstrated that the proposed extraction technique is a viable alternative for determination of imidacloprid in vegetables, soil and fruit samples.

\section{Acknowledgment}

The authors declare that there is no conflict of interests regarding the publication of this paper.

\section{References}

1. Vilchez JL, El-Khattabi R, Fernandez J, Gonzalez-Casado, Navalon A. Determination of imidacloprid in water and soil sample by gaschromatography-mass Spectrometry. J. Chrom. A. 1996;746(2):289294. doi:10.1016/0021-9673(96)00402-5.

2. Watanabe E, Eun H, Baba K, Arao T, Ishii Y, Endo S, Ueji M. Rapid and simple screening analysis for residual imidacloprid in agricultural products with commercially available ELISA. Analytica Chimica Acta. 2004;521(1):45-51. doi:10.1016/j.aca.2004.05.056.

3. Zhou Q, Ding Y, Xiao J. Sensitive determination of thiamethoxam, imidacloprid and acetamiprid in environmental water samples with solid-phase extraction packed with multiwalled carbon nanotubes prior to high-performance liquid chromatography. Anal Bioanal Chem. 2006;385(8):1520-5.

4. Baig SA, Akhter NA, Ashfaq M, Asi MR, Ashfaq U. Imidacloprid residues in vegetables, soil and water in the southern Punjab, Pakistan Journal of Agricultural Technology. 2012;8(3):903-916.

5. Srivastava Ashutosh K, Srivastava MK, Patel DK, Mudiam MKR Srivastava LP. Gas-Chromatographic Determination of Imidacloprid in Water. J. Environ. Res. and Development. 2012;7(2):643-651.

6. Samnani P, Vishwakarma K, Pandey SY. Simple and sensitive method for determination of imidacloprid residue in soil and water by HPLC. Bull Environ Contam Toxicol. 2011;86(5):554-8. doi: 10.1007/ s00128-011-0245-8.

7. Anastassiades M, Lehotay SJ, Stajnbaher D, Schenck FJ. Fast and easy multiresidue method employing acetonitrile extraction/partitioning and "Dispersive solid-phase extraction" For the determination of pesticide residues in produce. J AOAC Int. 2003;86(2):412-31.

8. Kapoor U, Srivastava MK, Srivastava AK, Patel DK, Garg V, Srivastava LP. Analysis of imidacloprid residues in fruits, vegetables, cereals, fruit juices, and baby foods, and daily intake estimation in and around Lucknow, India. Environ Toxicol Chem. 2013;32(3):723-7. doi: 10.1002/etc.2104.

9. R Schoning, R Schmuck. Analytical determination of imidacloprid and relevant metabolite residues by LC MS/MS. Bulletin of Insectology. 2003;56(1):41-50.

10. Method validation and quality control procedures for pesticide residues analysis in food and feed, DocumentNO. SANCO/10684/2009. SANCO;2009. 